\title{
Isolation and Quantitative Analysis of Chemical Constituents in Codonopsis lanceolata
}

\author{
Yeongdon $\mathrm{Ju}^{1,2, *}$, Jeong Wook Jeon ${ }^{3, * *}$ and Kyung-Yae Hyun ${ }^{4, \dagger, * * *}$ \\ ${ }^{I}$ Department of Clinical Laboratory Science, College of Health Sciences, \\ Catholic University of Pusan, Busan 46252, Korea \\ ${ }^{2}$ Clinical Trial Specialist Program for in Vito Diagnostics, Brain Busan 21 Plus Program, \\ the Graduate School, Catholic University of Pusan, Busan 46252, Korea \\ ${ }^{3}$ Nutricare Co Ltd, Gangwon-do 25250, Korea \\ ${ }^{4}$ Department of Clinical Laboratory Science, Dong-Eui University, Busan 47340, Korea
}

Codonopsis lanceolata has numerous chemical constituents that includes polyphenols, saponins, tannins, triterpene, alkaloids, and steroids. The extract of $C$. lanceolata was partitioned with Haxane, $\mathrm{CH}_{2} \mathrm{Cl}_{2}$, EtOAc, $n$ - $\mathrm{BuOH}$ and $\mathrm{MeOH}$. The determination of structure for lancemaside $\mathrm{G}$, lancemaside $\mathrm{B}$, lancemaside A were based on physicochemical and HPLC chromatogram data, including NMR and HR-MS. In addition, tangshenoside I and lobetyolin were identified in the material separation process for the extract of $C$. lanceolata, and content analysis was performed using HPLC. The compounds were confirmed as lancemaside $\mathrm{G}$, lancemaside $\mathrm{B}$, lancemaside A, tangshenoside I, and lobetyolin.

Key Words: Codonopsis lanceolata, Lancemaside G, Lancemaside B, Lancemaside A, Tangshenoside I, Lobetyolin

\section{서 론}

더덕(Codonopsis lanceolata)은 초롱꽃과에 속하는 다년 생 덩굴식물로 뿌리는 직근으로 땅속 깊이 들어 있어 형 상이 인삼, 도라지와 유사하며, 우리나라, 일본 및 중국 등에서 주로 약용과 식용으로 이용하고 있다(Choi and Choi, 1999; Kim et al., 2010). 우리나라의 경우 강원도 및 제 주도에서 주로 생산되고 있다(Kim et al., 2010). 천연물, 농 수산물 및 약용식물을 대상으로 생리 활성 평가 및 물질 분리에 대한 연구는 지속적으로 진행되어 왔고, 더덕을 대상으로 기능성 식품 및 화장품 등 다양한 목적으로 연 구들이 진행되었다(Shim and Chun, 2012; Chung et al., 2014). 더덕은 전통 의학에서 널리 사용되었으며 기관지염, 기침,
경련, 정신 신경증, 암, 비만, 고지혈증, 부종 간염, 대장 염 및 폐 손상과 같은 질병과 증상에 효과가 있다. 또한 polyphenols, saponins, tannins, triterpene, alkaloids, 및 steroids 등 다양한 생물학적 활성화합물을 지니고 있다(Hossen et al., 2016). 이 중 더덕의 saponin 성분은 강력한 항 염 증 작용을 보인다고 알려져 있다(Byeon et al., 2009). 더덕 의 saponin 은 lancemaside $\mathrm{A}$, lancemaside $\mathrm{B}$, lancemaside $\mathrm{C}$, lancemaside $\mathrm{E}$, lancemaside $\mathrm{G}$, foetidissimoside $\mathrm{A}$, aster saponin $\mathrm{Hb}$ 등이 있다. 이 중 lancemaside $\mathrm{A}$ 성분이 더덕 뿌리에 서 가장 풍부하게 존재한다고 알려져 있다(Shirota et al., 2008). 특히 더덕의 saponin 성분 중 lancemaside $\mathrm{A}$ 는 2,4,6trinitrobenzenesulfonic acid의 작용으로 대장염을 완화하고 항 염증 활성과 관련이 있다고 보고되었다(Lee et al., 2019; Kim et al., 2014). 따라서 본 연구에서 더덕 추출물의 주요

Received: August 13, 2021 / Revised: September 13, 2021 / Accepted: September 14, 2021

${ }^{*}$ Graduate student, ${ }^{* *}$ Researcher, ${ }^{* * *}$ Professor.

${ }^{\dagger}$ Corresponding author: Kyung-Yae Hyun. Department of Clinical Laboratory Science, Dong-Eui University, Busan 47340, Korea.

Tel: +82-51-890-2683, Fax:+82-0505-182-6877, e-mail: kyhyun@deu.ac.kr

(OThe Korean Society for Biomedical Laboratory Sciences. All rights reserved.

(9)This is an Open Access article distributed under the terms of the Creative Commons Attribution Non-Commercial License (http://creativecommons.org/licenses/by-nc/3.0/) which permits unrestricted non-commercial use, distribution, and reproduction in any medium, provided the original work is properly cited. 
한 성분인 saponin을 정제하여 지표성분의 함량 분석을 실시하여 더덕에서 유래한 생리활성물질에 대해 이용성 을 확인하는 기초자료를 확보하고자 한다.

\section{재료 및 방법}

\section{실험재료 및 시약}

본 연구에서 사용한 더덕은 강원도 동횡성농협에서 구 입한 더덕으로 (주)뉴트리케어에서 추출을 진행하였으며, 각각의 시료를 desiccator에 보관하여 사용하였다. 분석에 사용된 Distilled water, methanol, ethanol, acetic acid 등의 시 약은 HPLC (High Performance Liquid Chromatography) 등 급을 사용하였다.

\section{표준용액 제조}

Tanshenoside I 및 Lobetyolin 각각의 표준품 적정량을 정밀하게 달아 메탄올 $(\mathrm{MeOH})$ 로 용해하여 약 $1.0 \mathrm{mg} / \mathrm{mL}$ 가 되게 녹여 표준원액으로 하였다. 상기용액을 표준원액 (stock solution)으로 하며 메탄올로 희석하여 표준용액으로 사용하였다.

\section{시험용액 제조}

Tanshenoside I 및 Lobetyolin 각각의 시료 $50 \mathrm{mg}$ 을 칭량 한 후 $25 \mathrm{~mL}$ 부피의 플라스크에 넣어 $50 \%$ 에탄올 $(\mathrm{EtOH})$ 로 정용하고, 20 분 동안 초음파 추출을 진행한 후 $0.45 \mu \mathrm{m}$ syringe filter로 여과하여 HPLC 분석용 시료로 사용하였다.

\section{추출물 및 용매분획물 제조}

더덕 추출은 강원도 동횡성농협에서 구입한 3 년근 건 조 더덕 절편 $3.5 \mathrm{~kg}$ 을 $50 \%$ 주정 $20 \mathrm{~L}$ 사용하여 $50^{\circ} \mathrm{C}$ 에서 20 시간 추출을 진행하였다. 식품의약품안전처의 기능성 원료 표준화 지침서를 참조하여 최적의 추출용매 조건을 설정하였으며, 기능성분 및 지표성분을 분석하기 위하여 $50 \%$ 의 주정조건으로 설정하여 실험을 진행하였다. 추출 시간은 8 시간에서 24 시간 사이의 조건으로, 추출온도는 $20^{\circ} \mathrm{C}$ 에서 $80^{\circ} \mathrm{C}$ 사이의 조건으로 진행하였으며 그 결과 $50{ }^{\circ} \mathrm{C}$ 에서 20 시간의 조건으로 추출하였을 때 추출함량이 가장 높게 나타나 해당 조건으로 실험을 진행하였다. 또 한 감압건조를 실시하여 열풍건조의 조건으로 $50^{\circ} \mathrm{C}$ 에서 3 일간 건조한 후, 총 $104.3 \mathrm{~g}$ 의 조 추출물을 획득하였다. 추출물 용매분획은 분별 깔때기를 이용하여 진행하였다. 농축된 더덕 추출물 $(104.3 \mathrm{~g})$ 을 물 $(700 \mathrm{~mL})$ 에 녹인 후,

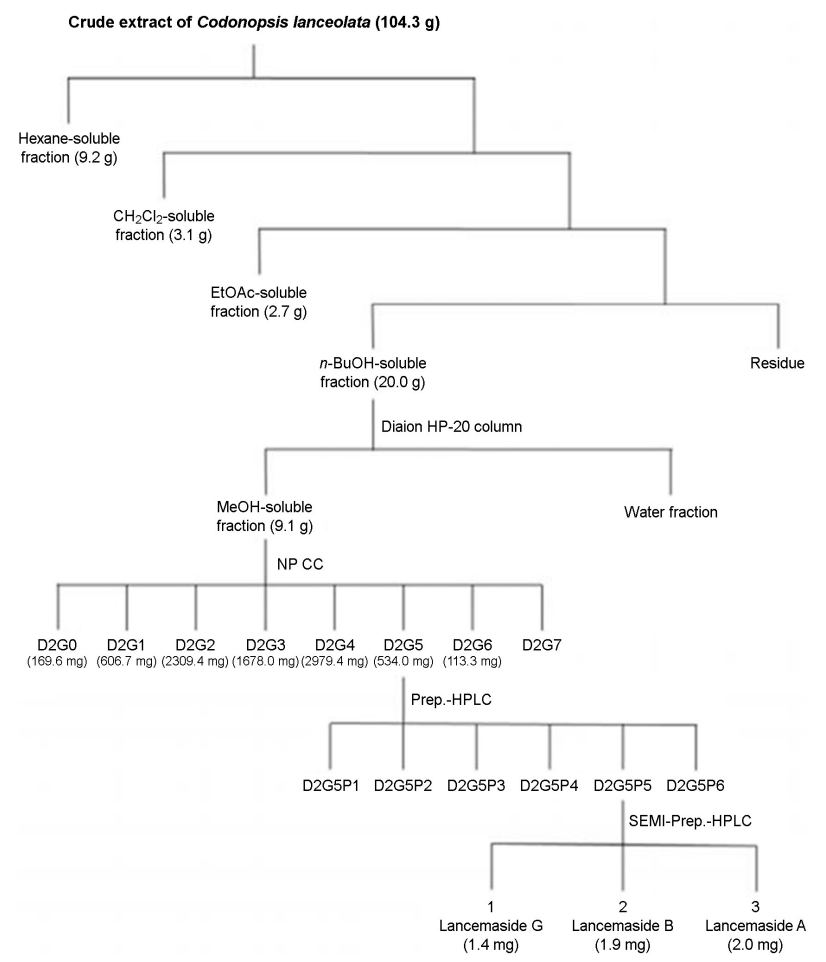

Fig. 1. Flow diagram the extract and fractionation of Codonopsis lanceolata. The concentrated Codonopsis lanceolata extract (104.3 g) was dissolved in water $(700 \mathrm{~mL})$. Then, fraction was carried out with hexane $(9.2 \mathrm{~g})$, methylene chloride (3.1 g), ethyl acetate $(2.7 \mathrm{~g})$, and butanol (20 g), respectively. The methanol fraction $(9.1 \mathrm{~g})$ was obtained using a Diaion HP-20 column.

헥산(Hexane), 메틸렌클로라이드 $\left(\mathrm{CH}_{2} \mathrm{Cl}_{2}\right)$, 에틸아세테이트 (Ethyl acetate, EtOAc), 부탄올 $(n-\mathrm{BuOH})$ 로 각각 용매분획을 실시하였다. 얻어진 추출물의 부탄올 분획 $(20.0 \mathrm{~g})$ 에서 당 을 제거하기 위해 Diaion HP-20을 이용하여 증류수로 당 을 제거하고, 메탄올 분획 $9.1 \mathrm{~g}$ 을 획득하였다(Fig. 1).

\section{HPLC 분석조건}

더덕 추출물에 함유되어 있는 tangshenoside I 및 lobetyolin 표준품을 HPLC로 분석한 검출시간(Retention time, $\mathrm{RT})$ 과 시료를 표준품과 동일한 조건으로 분석한 결과와 비교하여 동정하였고, 시료용액에 일정량의 표준품을 첨 가하여 동시에 HPLC에서 분석을 실시하여 검출된 peak의 모양 및 검출시간을 측정하여 확인하였다. Tangshenoside $\mathrm{I}$ 의 경우 16.87 분, Lobetyolin의 경우 18.95 분으로 나타났다. 또한 본 실험의 분석조건에서 표준물질의 분리 상태는 양 호하였고, 전 처리한 더덕 시료의 chromatogram을 비교하 여 각각의 성분의 peak가 분리되는지 확인한 결과 각 성 


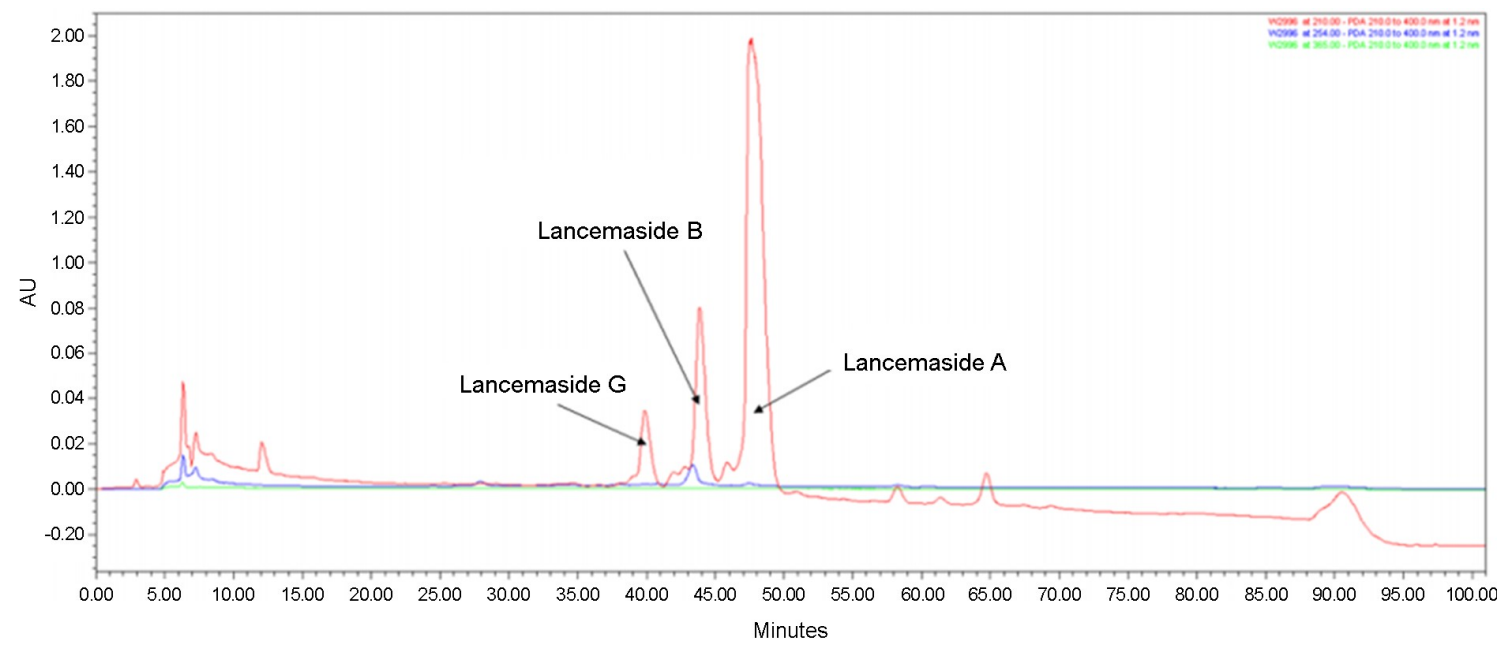

Fig. 2. HPLC chromatograms of three lancemaside compounds in Codonopsis lanceolata extracts. Compound 1: lancemaside G, Compound 2: lancemaside B, Compound 3: lancemaside A.

분들은 다른 peak와 간섭이 없이 분리되었음을 확인하였 고, 표준용액 peak의 검출시간과 더덕 추출물의 검출시간 이 일치하였다. 분석조건은 HPLC (Shimadzu HPLC system) 를 이용하였고, ACE $5(4.6 \times 250 \mathrm{~mm}, 5 \mu \mathrm{m})$ column을 사용 하였다. 이동상 용매는 $0.1 \%$ acetic acid(용매 A), acetonitrile (용매 B)를 사용하여 gradient를 주어 tangshenoside I과 lobetyolin을 동시에 분석하였다. 이동상 농도 구배조건은 용매 $\mathrm{B}$ 를 $5 \%$ 로 시작하여 3 분 동안 유지하였고, 17 분까 지 $95 \%$ 로 증가시킨 다음 3 분 동안 유지하다가, 22 분까 지 $5 \%$ 로 감소시키고 25 분까지 유지하였다. 용출 속도는 $0.5 \mathrm{~mL} / \mathrm{min}$, column의 온도는 $30^{\circ} \mathrm{C}$ 로 유지하였고, 검출기 는 UV detector, 검출 파장은 $267 \mathrm{~nm}$ 에서 측정하였다.

\section{결 과}

\section{$\mathrm{HR}-\mathrm{MS}$ 및 $\mathrm{HPLC}$ 분석}

메탄올 분획 $(9.1 \mathrm{~g})$ 을 Normal phase open column chromatography (EtOAc:MeOH:Water, 9:3:1)를 이용하여 8개의 분획(D2G0-D2G7)으로 나누었고, 분획 D2G5 $(534.0 \mathrm{mg})$ 을 Preparative HPLC $(40 \% \rightarrow 100 \% \mathrm{MeOH}$, gradient system) 를 이용하여 6개의 분획(D2G5P1-P6)으로 나누었다. High resolution electrospray ionization quadrupole time-of-flight mass spectrometer (HR-ESI-QTOF-MS) profile 분석을 통해, lancemaside 계통의 saponin 물질들이 분포되어 있는 것으 로 보이는 분획 D2G5P5을 확인하였으며, 해당 분획을 Semi-preparative HPLC $(55 \% \rightarrow 80 \% \mathrm{MeOH}$, gradient system)
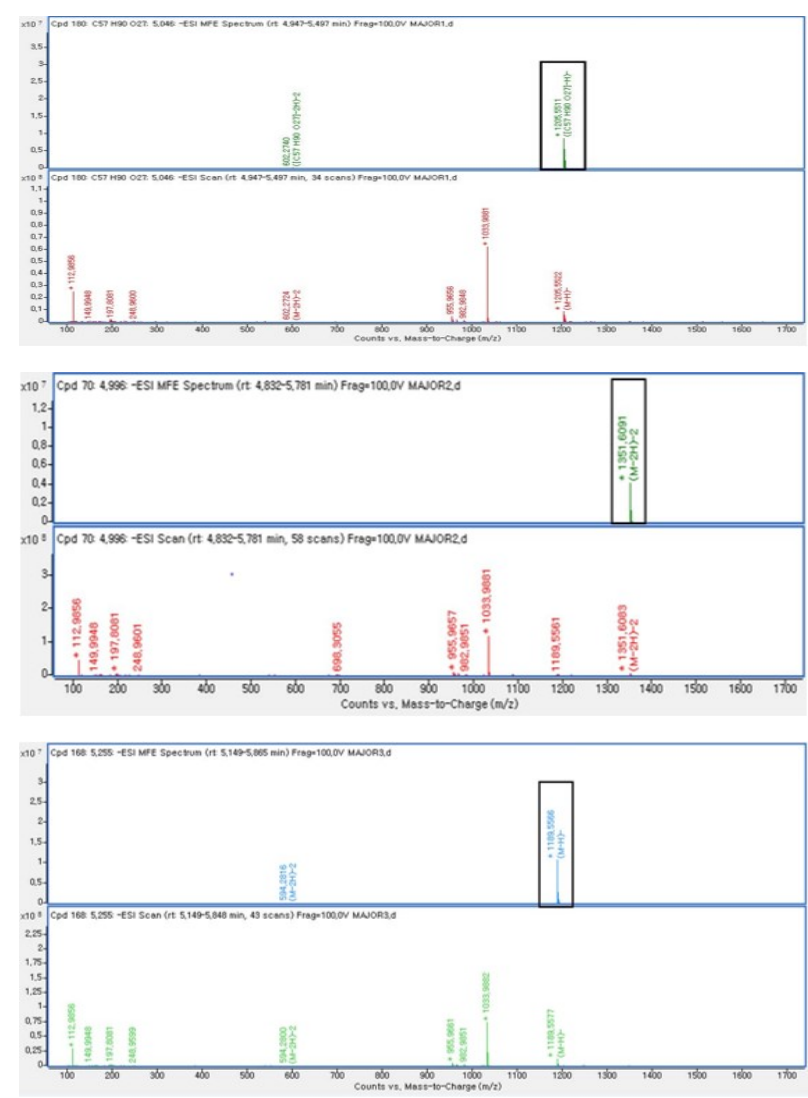

Fig. 3. HR-MS data of three lancemaside compounds in Codonopsis lanceolata extracts. Compound 1: lancemaside $G$ $\left(\mathrm{C}_{57} \mathrm{H}_{90} \mathrm{O}_{27}\right)$, Exact Mass; 1206.5586, m/z; $1205.5522[\mathrm{M}-\mathrm{H}]$; , Compound 2: lancemaside $\mathrm{B}\left(\mathrm{C}_{63} \mathrm{H}_{100} \mathrm{O}_{31}\right)$, Exact Mass; $1352.6249, \mathrm{~m} / z$; 1351.6091 [M-2H] $]^{--}$, Compound 3: lancemaside A $\left(\mathrm{C}_{57} \mathrm{H}_{90} \mathrm{O}_{26}\right)$, Exact Mass; $1190.5643, \mathrm{~m} / \mathrm{z} ; 1189.5566[\mathrm{M}-\mathrm{H}]^{-}$. 
를 이용하여 지표물질 1 (lancemaside G), 2 (lancemaside B), 3 (lancemaside A)을 분리하였다(Fig. 1, Fig. 2).

\section{성분 구조 동정}

더덕 추출물에서 총 3 종의 지표물질을 분리하였고 지표 물질의 여부를 알아보기 위해서 HR-ESI-QTOF-MS [10\% $\rightarrow 100 \%$ Acetonitrile $(\mathrm{MeCN})$, gradient system]를 사용하여
메탄올 분획에서 지표물질의 분자량이 있음을 확인하였 다. 또한 화학적 구조를 규명하기 위해 pyridine-d $\mathrm{d}_{5}$ 에 녹여 ${ }^{1} \mathrm{H}-\mathrm{NMR}$ (Nuclear magnetic resonance) 및 HR-MS (Highresolution mass spectrometer) data를 활용하여 결정하였다 (Table 1, Fig. 3). Electrospray ionization mass spectrum을 측 정한 결과 첫 번째 화합물은 $\mathrm{m} / \mathrm{z}$ 1205.5522에서 [M-H] 에 기인하는 peak를 관찰하여 분자량 1206.5586 을 확인

Table 1. ${ }^{1} \mathrm{H}(850 \mathrm{MHz})$ NMR data (pyridine- $\left.\mathrm{d}_{5}\right)$ of three compounds isolated from Codonopsis lanceolata

\begin{tabular}{|c|c|c|c|c|c|c|}
\hline \multirow{3}{*}{$\begin{array}{l}\text { Position } \\
\mathrm{H}-1\end{array}$} & \multirow{2}{*}{\multicolumn{2}{|c|}{$\begin{array}{l}\text { Compound } 1 \\
\delta_{\mathrm{H}}(J \text { in } \mathrm{Hz})\end{array}$}} & \multirow{2}{*}{\multicolumn{2}{|c|}{$\begin{array}{c}\text { Compound } 2 \\
\delta_{\mathrm{H}}(J \text { in } \mathrm{Hz})\end{array}$}} & \multirow{2}{*}{\multicolumn{2}{|c|}{$\begin{array}{c}\text { Compound } 3 \\
\delta_{\mathrm{H}}(J \text { in } \mathrm{Hz})\end{array}$}} \\
\hline & & & & & & \\
\hline & 2.20 & Overlap & 1.37 & Overlap & 1.40 & $\mathrm{~d}(12.0)$ \\
\hline \multirow{3}{*}{2} & 1.15 & s & 0.87 & Overlap & 0.87 & Overlap \\
\hline & 4.71, & s & 2.28 & Overlap & 2.23 & $\mathrm{~d}(11.0)$ \\
\hline & & & 1.86, & Overlap & 1.85, & Overlap \\
\hline 3 & 3.42 & Overlap & 3.40 & Overlap & 3.38 & dd (12.0) \\
\hline 5 & 0.97, & $\mathrm{d}(11.0)$ & 0.78 & $\mathrm{~d}(12.0)$ & 0.79, & $\mathrm{d}(12.0)$ \\
\hline 9 & 1.74, & Overlap & 1.73, & Overlap & 1.73, & $t(8.5)$ \\
\hline 12 & 5.58 & s & 5.64 & Overlap & 5.58 & br. s \\
\hline 16 & 5.26 & s & 5.27, & $\mathrm{s}$ & 5.26 & s \\
\hline 18 & 3.58 & $\mathrm{~d}(13.0)$ & 3.51, & Overlap & 3.56 & $\mathrm{~d}(14.5)$ \\
\hline \multirow[t]{2}{*}{19} & 2.77, & $t(13.0)$ & 2.77, & $t(13.0)$ & 2.76 & $t(13.5)$ \\
\hline & 1.34, & $\mathrm{s}$ & 1.33, & Overlap & 1.30 & br. d \\
\hline \multirow[t]{2}{*}{21} & 2.42 & t like & 2.43 & Overlap & 2.41, & $t(10.5)$ \\
\hline & 1.29, & Overlap & 1.30 & Overlap & 1.30 & Overlap \\
\hline \multirow[t]{2}{*}{22} & 2.31, & Overlap & 2.30 & Overlap & 2.30 & $\mathrm{t}$ \\
\hline & 2.20 & Overlap & 2.17, & Overlap & 2.18 & $\mathrm{t}$ \\
\hline 23 & 1.34, & $\mathrm{s}$ & 1.27, & $\mathrm{s}$ & 1.27 & $\mathrm{~s}$ \\
\hline 24 & 1.37, & s & 0.95, & s & 0.97, & s \\
\hline 25 & 1.50 & $\mathrm{~s}$ & 0.80 & $\mathrm{~s}$ & 0.81, & $\mathrm{s}$ \\
\hline 26 & 1.11, & $\mathrm{s}$ & 1.07, & $\mathrm{s}$ & 1.05, & $\mathrm{s}$ \\
\hline 27 & 1.82 & s & 1.82 & s & 1.81, & $\mathrm{s}$ \\
\hline 29 & 1.00, & s & 0.98 & $\mathrm{~s}$ & 1.00 & $\mathrm{~s}$ \\
\hline 30 & 1.15 & $\mathrm{~s}$ & 1.12 & $\mathrm{~s}$ & 1.13, & $\mathrm{s}$ \\
\hline glcA-1 & 5.02 & Overlap & 5.03 & Overlap & 5.02 & $\mathrm{~d}(7.5)$ \\
\hline 5 & 4.54 & $\mathrm{~d}(9.0)$ & 4.71, & Overlap & 4.69, & $\mathrm{d}(9.0)$ \\
\hline ara-1 & 6.51 , & s & 6.40 & Overlap & 6.49, & br. s \\
\hline rha-1 & 5.70 & $\mathrm{~s}$ & 5.84 & $\mathrm{~s}$ & 5.69, & br. s \\
\hline 6 & 1.70, & s & 1.71, & $\mathrm{d}(6.0)$ & 1.70 & br. s \\
\hline xyl-1 & 5.18 & $\mathrm{~d}(7.5)$ & 5.15 & $\mathrm{~d}(7.0)$ & 5.17 & $\mathrm{~d}(7.5)$ \\
\hline xyl-1' & 5.23, & $\mathrm{d}(7.5)$ & 5.21, & $\mathrm{d}(7.5)$ & 5.22 & $\mathrm{~d}(7.5)$ \\
\hline glc-1 & & & 5.12 & $\mathrm{~d}(7.5)$ & & \\
\hline
\end{tabular}

Reference; Ushijima et al. Triterpene glycosides from the roots of Codonopsis lanceolata, 2008 


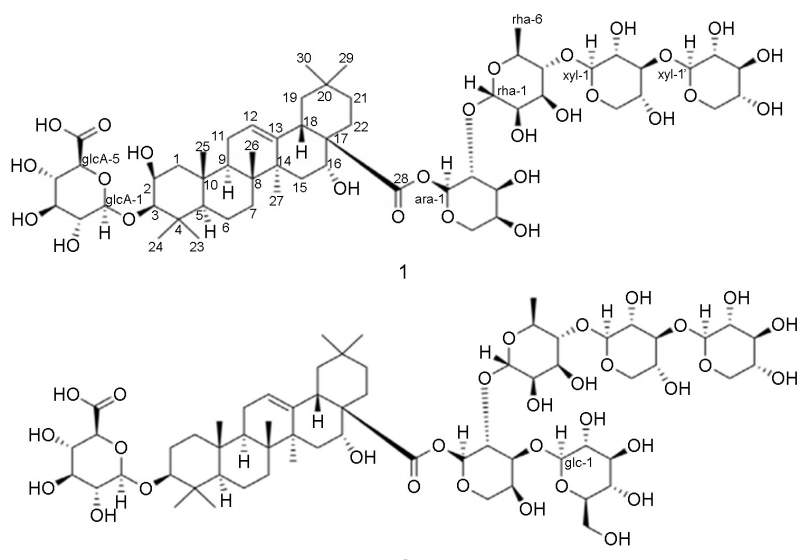

2

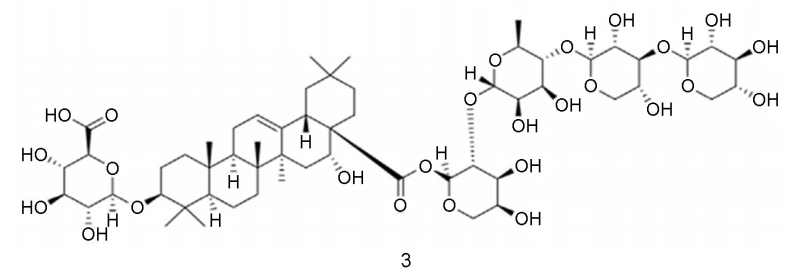

Fig. 4. Schematic diagram of the proposed fragmentation of three compounds in Codonopsis lanceolata extracts. 1: lancemaside G, 2: lancemaside B, 3: lancemaside A.
할 수 있었다. 두 번째 화합물은 $m / z$ 1351.6091에서 [M$2 \mathrm{H}]^{2}$ 에 기인하는 peak를 통해 분자량 1352.6249 를 확인하 였고, 세 번째 화합물은 $m / z 1189.5566$ 에서 [M-H]에 기인 하는 peak를 관찰, 분자량 1190.5643 을 확인할 수 있었다. 따라서 분리한 3 개의 화합물은 lancemaside $\mathrm{G}$ lancemaside $\mathrm{B}$, lancemaside A로 동정하였다(Fig. 4). 또한 Saponin 계열 lancemaside 3 종 화합물의 분리량은 lancemaside $\mathrm{G}$ 의 경우 $1.4 \mathrm{mg}$, lancemaside B는 $1.9 \mathrm{mg}$, lancemaside A는 $2.0 \mathrm{mg}$ 으 로 나타났으며 lancemaside 계통 물질들 중 lancemaside A 의 분리량이 가장 많은 것을 확인하였다.

\section{함량 분석}

더덕 추출물의 물질분리 과정에서 주요한 peak 2 개가 검출되었고, 각각 tangshenoside I, lobetyolin으로 확인하였 다(Fig. 5). 따라서 더덕의 tangshenoside I 및 lobetyolin의 함량을 확인하기 위해 분석을 진행하였다. 본 연구의 실 험값은 3 회 반복하였고, 기기 분석을 통해 산출한 함량 값을 기준으로 평균 및 표준편차를 나타내었다(Table 2). 또한 더덕의 주요한 성분 분석을 위해 분석법에 대해 직

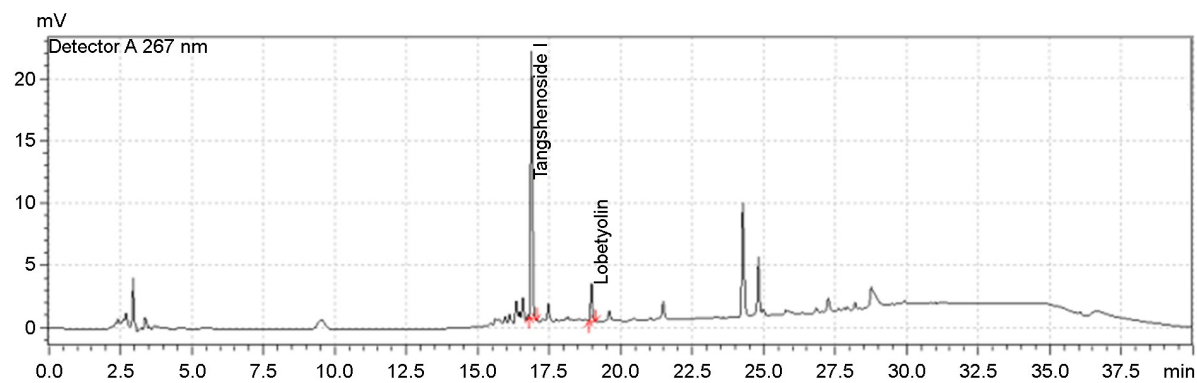

Fig. 5. HPLC chromatogram of tangshenoside $I$ and lobetyolin in powder of Codonopsis lanceolata extracts. Absorbance: $267 \mathrm{~nm}$.

Table 2. The contents of tangshenoside I and lobetyolin in Codonopsis lanceolata powder

\begin{tabular}{lcccc}
\hline \hline \multirow{2}{*}{ Compound } & No. & \multicolumn{3}{c}{ Mean \pm S.D. ${ }^{1)}(\mathrm{mg} / \mathrm{g}$, dry weight) } \\
\cline { 3 - 5 } & & Tangshenoside I & Lobetyolin & Total \\
\hline \multirow{3}{*}{ Codonopsis lanceolata powder } & 1 & $3.65 \pm 0.04$ & $1.56 \pm 0.02$ & $5.21 \pm 0.06$ \\
& 2 & $3.49 \pm 0.00$ & $1.50 \pm 0.00$ & $4.99 \pm 0.00$ \\
& 3 & $3.74 \pm 0.03$ & $1.60 \pm 0.02$ & $5.34 \pm 0.04$ \\
\hline
\end{tabular}

\footnotetext{
${ }^{1)}$ Mean \pm S.D. in triplicate $(n=3)$
}

Table 3. Preparation of calibration curve for tangshenoside I and lobetyolin

\begin{tabular}{cccc}
\hline \hline Compound & Range & Regression equation & $\mathrm{R}^{2}$ \\
\hline Tangshenoside I & $0.78 \sim 24.93$ & $\mathrm{y}=11830 \mathrm{x}+1045.4$ & 1 \\
Lobetyolin & $0.88 \sim 28.27$ & $\mathrm{y}=3924.7 \mathrm{x}+677.28$ & 1 \\
\hline
\end{tabular}


선성(linearity)을 확인하였다. 직선성을 평가하기 위해 6단 계의 농도 $(0.78 \sim 28.27 \mathrm{ppm})$ 표준품을 분석하였고, 이를 통 해 얻은 chromatogram에서 peak의 면적과 농도 간의 상관 관계로 검량선을 작성하였다(Table 3). 모든 검량선은 해당 농도 범위에서 높은 상관계수를 나타내었다 $\left(\mathrm{R}^{2}=1\right)$.

\section{고 찰}

본 연구는 더덕의 주요 성분인 saponin의 정제 및 유효 성분의 함량 분석을 통해 더덕 유래 생리활성물질 및 이 용성 증진 연구의 기초자료를 확보하고자 하였다. 더덕 추출물에서 총 3종의 화합물을 분리할 수 있었고, 분리한 3 개의 화합물은 lancemaside G, lancemaside B, lancemaside $\mathrm{A}$ 로 동정하였다. 특히, 화합물 lancemaside $\mathrm{A}$ 는 더덕에서 분리한 saponin 계통 lancemaside 물질들 중 가장 많은 양 을 지닌 것으로 나타났다. 또한 더덕 추출물의 물질분리 과정에서 tangshenoside I 및 lobetyolin을 더덕에서 많은 양 을 차지하고 있는 주요 화합물로 확인하였으며, HPLC를 통해 tangshenoside I 및 lobetyolin을 동시 분석하고, 더덕 분말의 tangshenoside I 및 lobetyolin의 함량이 각각 3.49 $3.74 \mathrm{mg} / \mathrm{g}, 1.50 \sim 1.60 \mathrm{mg} / \mathrm{g}$ 으로 나타난 것을 확인하였다. 더덕에서 분리한 saponin 계열 lancemaside 화합물 중 가 장 많은 양으로 확인된 lancemaside $\mathrm{A}$ 의 경우 염증의 조 절 및 억제에 관한 연구들이 활발하게 이루어지고 있다 (Kim et al., 2014; Joh and Kim, 2010). 뿐만 아니라 심혈관계 질환 개선 효과를 확인하여 이를 바탕으로 건강기능식품 을 개발하는 연구도 진행 중이다(Han et al., 2018; Shin et al., 2019). 실험에서 확인한 tangshenoside I 및 lobetyolin은 Codonopsis 속 식물의 주요한 화합물로, Codonopsis 속의 지표성분으로 선정한 연구결과 및 우리나라 지역별 더덕 의 tangshenoside 및 lobetyolin을 정량 분석한 연구결과를 통해 더덕의 지표물질 사용 가능성을 확인할 수 있었다 (Ichikawa et al., 2009; Hwang et al., 2018). 하지만 더덕의 tangshenoside I 및 lobetyolin을 이용한 기능성 연구는 다른 화합물에 비해 다양하고 많은 연구가 이루어지지 않고 있어 더덕에서 추출한 tangshenoside I 및 lobetyolin을 기능 성분으로 사용하기 위해서는 추가적인 실험이 필요한 상 황이다. 따라서 본 연구의 결과를 통해 더덕의 생리활성 물질 및 이용성 증진 연구를 지속하는데 기초자료로써 활용될 수 있을 것으로 사료된다.

\section{ACKNOWLEDGEMENT}

This research was supported by 2020 Regional Specialized Industry Promotion (Non-R\&D) Project of Hongcheon Institute of Medicinal Herb (HIMH) and Brain Busan 21 Plus (BB21+) Project.

\section{CONFLICT OF INTEREST}

The authors declare that they have no conflict of interest.

\section{REFERENCES}

Byeon SE, Choi WS, Hong EK, Lee J, Rhee MH, Park HJ, et al. Inhibitory effect of saponin fraction from Codonopsis lanceolata on immune cell-mediated inflammatory responses. Arch Pharm Res. 2009. 32: 813-822.

Choi MS, Choi PS. Plant Regeneration and Saponin Contents in Codonopsis lanceolata L.. Korean J Med Crop Sci. 1999. 7 : 275-281.

Chung KH, Jo HJ, Yoon JA, Song BC, An JH. Free Radicalscavenging Activities of Amaranth (Amaranthus spp. L.) Seed Extracts. Food Eng Prog. 2014. 18: 116-123.

Han AY, Lee YS, Kwon SH, Lee HS, Lee KW, Seol GH. Codonopsis lanceolata extract prevents hypertension in rats. Phytomedicine. 2018. 15: 119-124.

Hossen MJ, Kim MY, Kim JH, Cho JY. Codonopsis lanceolata: A Review of Its Therapeutic Potentials. Phytother Res. 2016. 30: 347-356.

Hwang BS, Kim JY, Jang M, Kim GC, Park YH, Hwang IG. Quantitative Analysis of Tangshenoside I and Lobetyolin from Korean Deoduk (Codonopsis lanceolata). Korean J. Food Nutr. 2018. 31: 957-963.

Ichikawa M, Ohta S, Komoto N, Ushijima M, Kodera Y, Hayama $\mathrm{M}$, et al. Simultaneous determination of seven saponins in the roots of Codonopsis lanceolata by liquid chromatographymass spectrometry. J Nat Med. 2009. 63: 52-57.

Joh EH, Kim DH. Lancemaside A inhibits lipopolysaccharideinduced inflammation by targeting LPS/TLR4 complex. J Cell Biochem. 2010. 111: 865-871.

Kim E, Yang WS, Kim JH, Park JG, Kim HG, Ko J, et al. Lancemaside A from Codonopsis lanceolata modulates the inflammatory responses mediated by monocytes and macrophages. Mediators Inflamm. 2014. 2014: 405158.

Kim JA, Moon HK, Choi YE. Triterpenoid Saponin Contents of the Leaf, Stem and Root of Codonopsis lanceolata. Korean J 
Med Crop Sci. 2014. 22: 1-7.

Kim NY, Chae HS, Lee IS, Kim DS, Seo KT, Park SJ. Analysis of Chemical Composition and Antioxidant Activity of Codonopsis lanceolata Skin. J Korean Soc Food Sci Nutr. 2010. 11: 16271633.

Kim SS, Jeong MH, Seo YC, Kim JS, Kim NS, Woon WB, et al. Comparison of Antioxidant Activities by High Pressure Extraction of Codonopsis lanceolata from Different Production Areas. Korean J Med Crop Sci. 2010. 18: 248-254.

Lee MJ, Nam JH, Um IE, Kang CK, Rho IR. Determination the optimum extraction method for saponin lancemasides in Codonopsis lanceolata. Korean J Food Sci Technol. 2019. 51: 103-108.

Shim SB, Chun YJ. The Study on Skin Safety and Efficacy of Codonopsis lanceolata Root Fermentation Extract. JKAIS. 2012. 11: 5623-5627.

Shin YK, Han AY, Hsieh YS, Kwon SH, Kim JH, Lee KW, et al. Lancemaside A from Codonopsis lanceolata prevents hyper- tension by inhibiting NADPH oxidase 2-mediated MAPK signalling and improving NO bioavailability in rats. J Pharm Pharmacol. 2019. 71: 1458-1468.

Shirota O, Nagamatsu K, Sekita S, Komoto N, Kuroyanagi M, Ichikawa $\mathrm{M}$, et al. Preparative separation of the saponin Lancemaside a from Codonopsis lanceolata by centrifugal partition chromatography. Phytochem Anal. 2008. 19: 403-410.

Ushijima M, Komoto N, Sugizono Y, Mizuno I, Sumihiro M, Ichikawa $\mathrm{M}$, et al. Triterpene glycosides from the roots of Codonopsis lanceolata. Chem Pharm Bull. 2008. 56: 308-314.

https://doi.org/10.15616/BSL.2021.27.3.154

Cite this article as: $\mathrm{Ju}$ Y, Jeon JW, Hyun KY. Isolation and Quantitative Analysis of Chemical Constituents in Codonopsis lanceolata. Biomedical Science Letters. 2021. 27: 154-160. 